\title{
Shear-Induced Orientation Transitions in Triblock Copolymer Styrene-Butadiene-Styrene with Cylindrical Domain Morphology
}

\author{
Diane B. Scott, Alan J. Waddon, Ye-Gang Lin, Frank E. Karasz," and \\ H. Henning Winter*
}

Department of Chemical Engineering and Department of Polymer Science and Engineering, University of Massachusetts, Amherst, Massachusetts 01003

Received August 26, 1991; Revised Manuscript Received March 31, 1992

\begin{abstract}
Shear-induced alignment of phase-separated domains in a triblock copolymer has been studied by transmission electron microscopy (TEM) and small-angle X-ray scattering (SAXS). The material used was triblock copolymer styrene-butadiene-styrene with a phase-separated morphology of hexagonally packed cylinders of poly(styrene) in a poly(butadiene) matrix. We started with an ordered structure, where the cylinders were aligned with a common director throughout the sample, and imposed a well-defined shear flow perpendicular to this director. Shear flow rotated the cylinder orientation by $90^{\circ}$ into the shear direction. At all observed strains, the hexagonal faces preferred to orient parallel to the shear plane. To produce the initial order, a solution-cast film was subjected to planar extension, aligning the cylinders in the extension direction. With a novel sample preparation technique, we were able to transform this rectangular sample into an axisymmetric geometry needed for large shear strain in a rotational rheometer with parallel disk fixtures. SAXS and TEM were used to observe shear-induced orientation transitions as a function of applied strain on room-temperature-quenched samples. We observed intermediate textures at small strains, maximum alignment at intermediate strains, and deterioration of order at large strains. By following the transitions from one aligned structure to another, we discuss the processes that produce domain orientation under shear flow.
\end{abstract}

\section{Introduction}

Triblock copolymer styrene-butadiene-styrene is a microphase-separated polymer that has gained widespread use as a thermoplastic elastomer. For high molecular weight and at low temperatures, the blocks are thermodynamically incompatible, causing the system to phase separate into domains rich in either one of the components. The blocks are covalently bonded together, restricting the phase separation to the order of the radii of gyration of the copolymer blocks, usually on the order of $100 \AA$. The equilibrium-phase-separated morphology depends primarily on the bulk polymer composition. ${ }^{1}$ Depending on the volume fraction of each block present, the two-phase structure may be periodically arranged spherical or cylindrical domains of one component in a continuous three-dimensional matrix of the second component or a two-dimensional lamellar structure in which the two components alternate. Theoretically, the domain spacings are found to scale as a fractional power of the molecular weight. ${ }^{2}$ The performance and flow properties of these block copolymers are strongly dependent on the material's morphology.

Processing of block copolymers may produce global orientation of the phase-separated domains on a macroscopic scale, referred to as a "single crystal" type structure. The Bristol group was the first to observe a flow-induced single crystal structure. These workers obtained crystalline-like X-ray diffraction patterns from extruded crumbs of KRATON 102, SBS copolymer. ${ }^{3-7}$ Several other researchers have since identified other conditions which produce single crystal structure such as oscillatory shear flow ${ }^{8-11}$ and steady shear ${ }^{12,13}$ in styrene block copolymers with cylindrical domain morphology. It is well established that shearing can produce global domain orientation, yet there is still no general understanding of the flow mechanisms.

This study is concerned with the progression of domain orientation in an SBS copolymer, with hexagonally packed cylindrical domains of PS in a PB matrix, during the early stages of shear flow. The order is characterized by the average direction of the cylindrical domains, the average direction of the lattice planes, and the fluctuations around these directions (degree of order). In order to study shearinduced domain orientation we first produced a highly ordered sample and sheared it normal to its initial direction of order. Well-defined shear strains were imposed on SBS in the molten state followed by rapid cooling to room temperature for morphological studies. Small-angle X-ray scattering (SAXS) and transmission electron microscopy (TEM) were used to detect the shear-induced structural transitions as a function of applied strain.

\section{Experimental Study}

Materials and Sample Preparation. The polymer used was a triblock copolymer SBS (Kraton 1102, Shell Development Company) containing $26 \mathrm{wt} \%$ polystyrene with molecular weight proportions of PS (10 600)-PB (60 100)-PS (10 600). Referring to its molecular weight, we will call it SBS81 throughout this text. The equilibrium-phase-separated morphology consists of hexagonally packed cylinders of poly(styrene) (PS) in a poly(butadiene) (PB) matrix. The PS is well below and the PB is well above their respective entanglement molecular weight. At all accessible temperatures, SBS81 is strongly phase separated. For cleaning, the polymer was twice precipitated into 2-propanol from a $10 \%$ toluene solution. Films were cast from a 10 wt $\%$ toluene solution with 0.5 wt \% Irganox as an antioxidant and vacuum-dried for $24 \mathrm{~h}$ at $150^{\circ} \mathrm{C} .12$

Experimental Procedure. The principle of our approach was to start with an initially ordered structure, where the cylinders were all aligned with a common director throughout the sample. To alter the alignment direction by $90^{\circ}$, a well-defined shear flow was applied with the shear direction perpendicular to the original director.

The initial orientation was produced by planar extension. This is a nonaxisymmetric, shear free flow in which the sample is compressed in one direction, elongated in the second direction (extension direction), and held at a constant width in the third direction. Rectangular films were cut from an as-cast film and stacked between two lubricated metal plates of a specially designed flow cell ${ }^{14-16}$ (which was placed in a Carver laboratory press), equilibrated at $120^{\circ} \mathrm{C}$, compressed to one-sixth of the initial height and annealed for $2 \mathrm{~h}$. The annealing step is essential for perfecting the domain structure. This technique gave an 
Sample Preparation

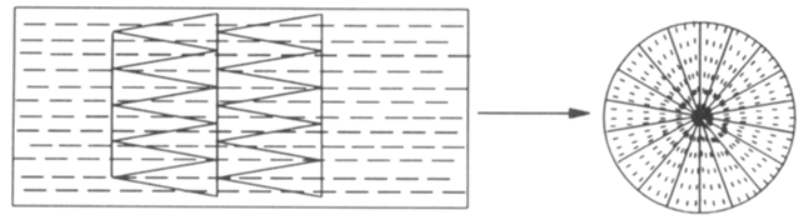

Experiment

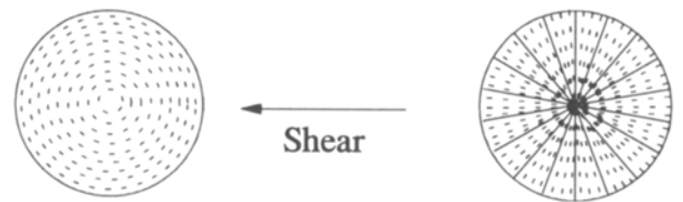

Figure 1. Schematic of special sample preparation and shear experiment. The planar extension orients the PS cylinders in the extension direction within a rectangular sheet from which a circular sample is pieced together and sheared. The dashed lines represent the local direction of PS domains. Real samples show a fluctuation of the director (see X-ray pattern, Figure 3).

ordered rectangular sheet with cylindrical domains aligned in the extension direction and hexagon planes parallel to the sample surface.

The rectangular sheet from planar extension does not suit the axisymmetric geometry required for rotational shearing experiments. A special sample preparation was required to radially align the cylinders. For this purpose, the ordered films were cut in an alternating zigzag pattern with respect to the cylinder orientation. A total number of 18 triangularly cut pieces were reconstructed to form a disk-shaped sample (like pieces of a pie) as shown schematically in Figure $1 .{ }^{14}$ The cut segments were mended together with toluene, a common solvent for both phases. The zigzag pattern was cut with an apex angle of $20^{\circ}$, giving a root-mean-squared error of $5.8^{\circ}$ in the cylinder misalignment.

The disk-shaped samples were subjected to an axisymmetric shear flow at a constant rate ( $\dot{\gamma}_{R}=0.01 / \mathrm{s}$ at the outer edge) using a Rheometrics mechanical spectrometer Model 800 in parallel plate geometry at $140{ }^{\circ} \mathrm{C}$. The direction of shear was circumferential, perpendicular to the initial cylinder orientation. The shear strain, $\gamma$, of a material element at a radial position, $r$, is given by

$$
\gamma=\theta r / H
$$

where $\theta$ is the angular displacement of the rotating plate in radians and $H$ is the sample height. Thus, with one sample we were able to study a range of applied strains by looking at different radial positions. Samples of $25-\mathrm{mm}$ diameter were subjected to shear strains at the outer edge $(r=R)$ of $\gamma_{R}=3$ and 13. These correspond to angular displacements of 14 and $60^{\circ}$, respectively. The displacement was held constant during rapid cooling to room temperature.

SAXS patterns were taken on film using a pinhole collimated Rigaku-Denki camera operating with a Ni-filtered $\mathrm{Cu} \mathrm{K} \alpha$ sealedtube radiation source at a sample to film distance of $243 \mathrm{~mm}$. A series of pictures with the X-ray beam parallel to all three axes of the sheared sample gave a full textural characterization. For TEM, thin samples were cut with a AO/Reichert FC 4 cryomicrotome using a diamond knife, both at $-110^{\circ} \mathrm{C}$. The $\mathrm{PB}$ phase was selectively stained with osmium tetraoxide to provide mass contrast between the phases for viewing in a JEOL $100 \mathrm{CX}$ electron microscope operating at $100 \mathrm{kV}$.

\section{Results}

Initial Morphology. The equilibrium-phase-separated morphology, after solution casting and vacuum drying, consists of hexagonally packed cylinders (domains) of PS, in a PB matrix. The molecules within the domains are disordered, except near the interface. On a micron scale, PS domains order within a grain, with a common director,

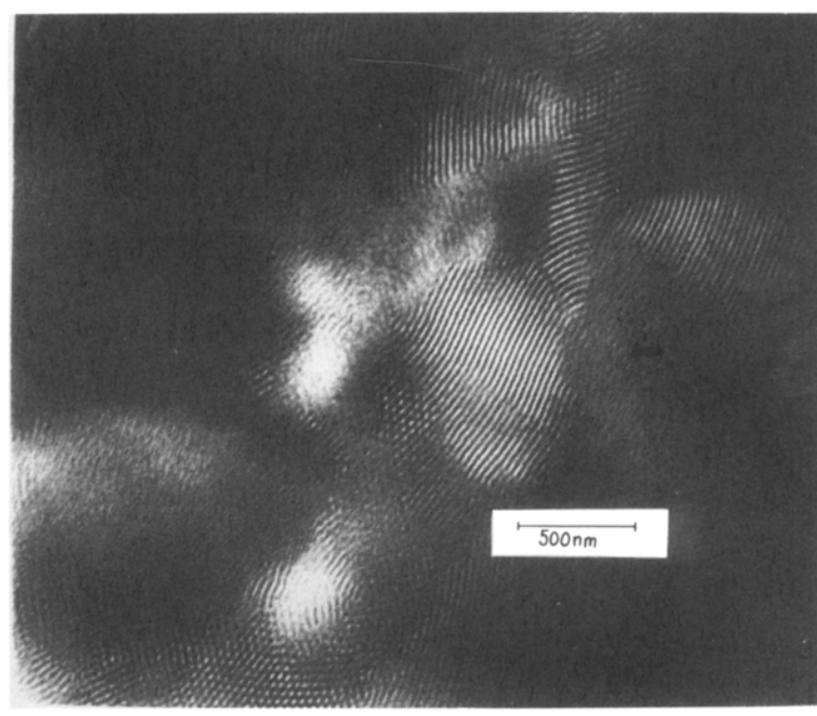

(a)

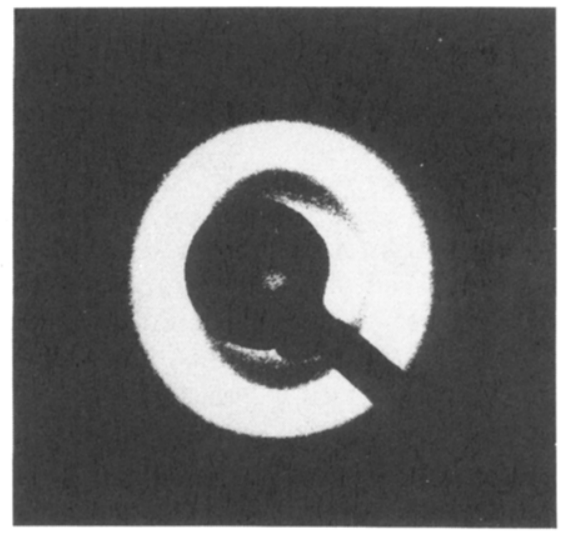

(b)

Figure 2. (a) Electron micrograph of as-cast SBS81 where the white regions are the cylindrical PS domains. (b) SAXS pattern of as-cast SBS81.

producing local anisotropy. Granular structure can be seen in the electron micrograph of Figure 2a. The director varies randomly from grain to grain giving a macroscopically isotropic sample, clearly demonstrated in the SAXS pattern of Figure $2 b$.

SAXS patterns of the sample after planar extension and before shear are shown in Figure 3. The patterns show reflections at 254,147 , and $96 \AA$, although only the inner-most reflection is visible here. Using Miller-Bravais notation these reflections correspond to the $\{10 \overline{1} 0\}$, $\{2 \overline{1} \overline{1} 0\}$, and $\{3 \overline{1} \overline{2} 0\}$ planes, respectively. In each case the value of the scattering angle is less than $1^{\circ}$. Therefore, a plane will be in a reflecting position when it is essentially parallel to the incident beam.

The two patterns taken with the beam perpendicular to the direction of orientation show only equatorial scattering, indicating that orientation of the cylinders is parallel to the direction of extensional flow (Figure 3a,c). However, comparison of these two patterns also reveals that the degree of arcing in the reflections is greater when the sample is viewed from the top (a) than when the beam is edge-on (b). This clearly suggests less order within the sample plane than at right angles to this plane. Figure $3 \mathrm{~b}$, with the beam in the direction of flow, shows a six-spot pattern from the $\{10 \overline{1} 0\}$ planes, corresponding to the 

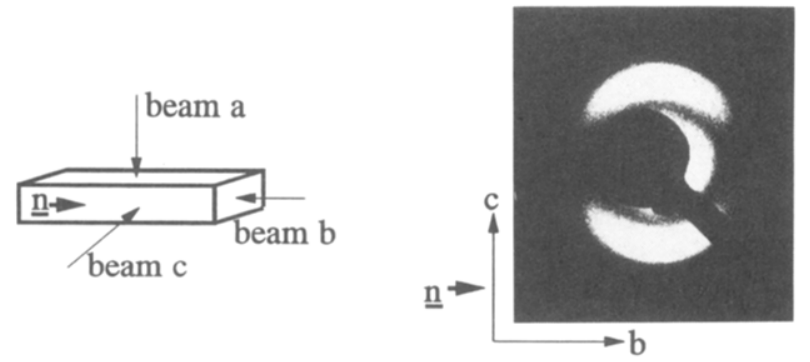

a) beam a
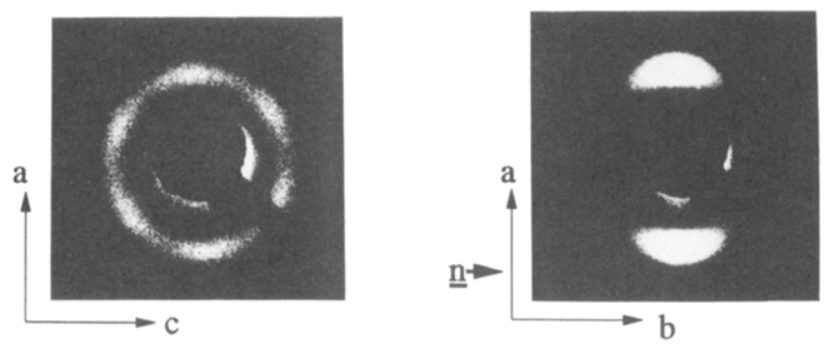

b) beam b

c) beam c

Figure 3. SAXS pattern of SBS81 after planar extension with an incident beam (a) normal to the flat sample, (b) in direction of extension, and (c) edge-on to the sample. n represents cylinder orientation.

(a)

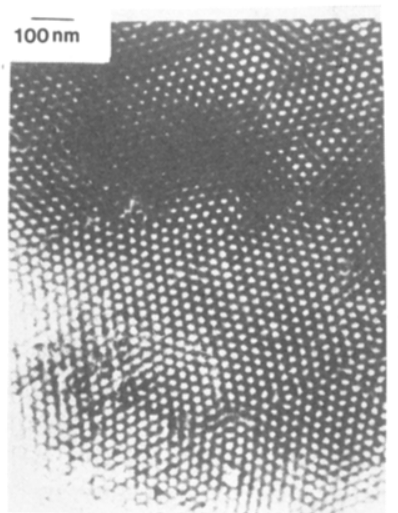

(b)

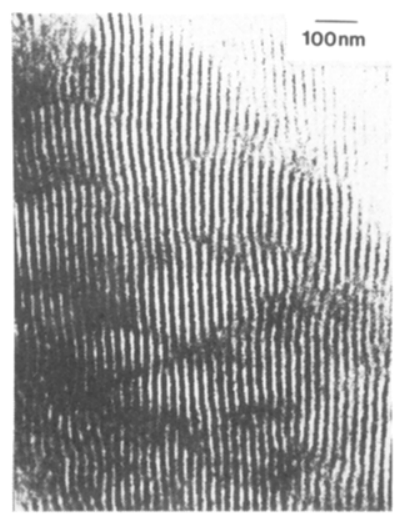

Figure 4. Electron micrograph of SBS81 after planar extension with a stretch $l / l_{\mathrm{o}}=6$, sectioned (a) perpendicular to extension and (b) normal to the $c$ direction.

reflections from the faces of the hexagons, although there is still significant disorder around this direction. Figure 3 is consistent with cylinders lying essentially parallel to the flow direction.

Also important is the alignment of the hexagon planes. We note that in Figure 3b there are positions of strong $\{10 \overline{1} 0\}$ intensity exactly above and below the sample plane, indicating a tendency for the cylinders to pack with the hexagon faces parallel to the sample boundary.

Figure 4a,b shows transmission electron micrographs of the ordered sample sectioned perpendicularand parallel to the direction of planar extension, respectively. These micrographs are completely consistent with the above SAXS patterns, again showing the PS cylinders oriented parallel to the direction of extension. However, even in this highly oriented sample grain boundaries are apparent; i.e. there are regions in which the "perfect" hexagonal packing is interrupted.

Shear-Induced Morphology. During this study, we have obtained numerous patterns over a range of applied

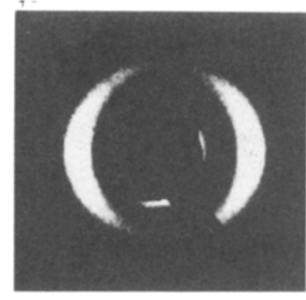

a) $\gamma=1.2 ; \gamma_{\mathrm{R}}=3$

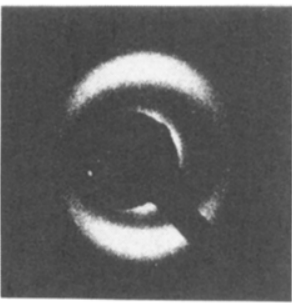

c) $\gamma=7.8 ; \gamma_{R}=13$

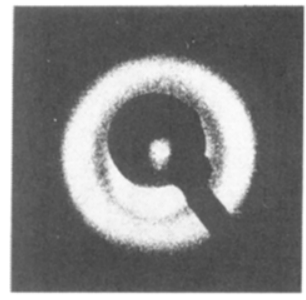

b) $\gamma=2.8 ; \gamma_{R}=3$
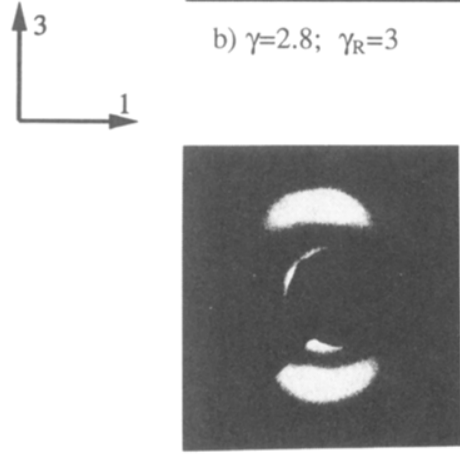

d) $\gamma=12 ; \gamma_{R}=13$
Figure 5. SAXS pattern with the incident beam parallel to the 2 axis, the sample normal. Each pattern is labeled with (1) the actual shear strain, $\gamma$, of the material element from which the SAXS pattern is taken and (2) the maximum shear strain, $\gamma_{R}$, at the outer edge of the sample $(r=R)$.

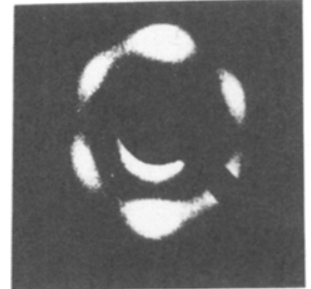

a) $\gamma=1.2 ; \gamma_{R}=3$

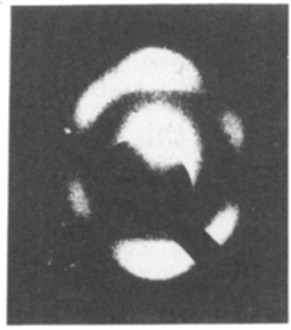

c) $\gamma=7.8 ; \gamma_{R}=13$

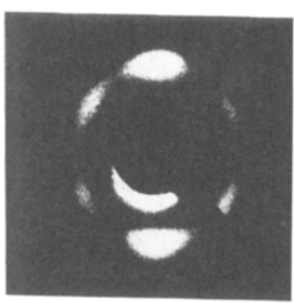

b) $\gamma=2.8 ; \quad \gamma_{R}=3$
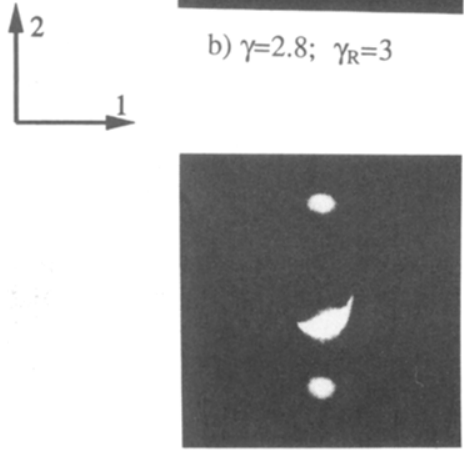

d) $\gamma=12 ; \gamma_{R}=13$
Figure 6. SAXS pattern with the incident beam parallel to the 3 axis, the sample radius.

strains of the sheared circular samples. For brevity, we only present the most representative and informative patterns. Figures 5-7 show a series of patterns taken at different radial positions on the sheared circular samples with the beam parallel to three directions. These three axes are conventionally referred to as axis 1 for the shear direction, axis 2 for the shear gradient direction, and axis 3 for the neutral direction (vorticity direction). For the axisymmetric sample geometry, these axes correspond to tangent, normal, and radius, respectively.

The imposed shear strain increases linearly with the radial position of a material element and provides a convenient way of sampling across a range of shear strains. Clearly, the patterns in all three directions show progresive changes with increasing amount of shear strain. Consider first the set of patterns with the beam parallel to the 2 axis, the sample normal (Figure $5 \mathrm{a}-\mathrm{d}$ ). At first, 


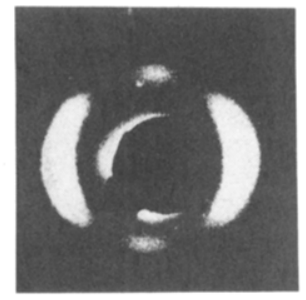

a) $\gamma=1.2 ; \gamma_{\mathrm{R}}=3$

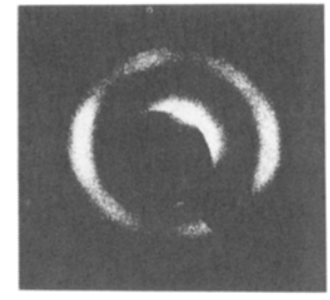

c) $\gamma=7.8 ; \gamma_{R}=13$

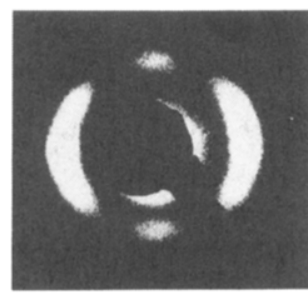

b) $\gamma=2.8 ; \gamma_{R}=3$ $4^{3}$

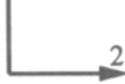
2

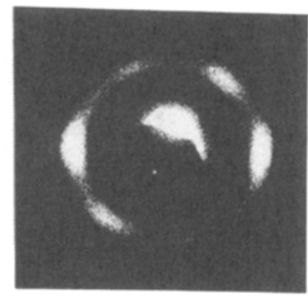

d) $\gamma=12 ; \gamma_{\mathrm{R}}=13$

Figure 7. SAXS pattern with the incident beam parallel to the 1 axis, the shear direction.

the original two-point pattern becomes progressively more disordered with increasing amounts of shear strain. At about $\gamma=2.8$ the pattern appears almost isotropic (Figure $5 \mathrm{~b})$. With further shear strain, the pattern once again becomes two point in character but with the maxima now located at $90^{\circ}$ to their original position in the unsheared sample (Figure 5c). With further shear strain, the maxima retain their position but become increasingly sharp (Figure $5 \mathrm{~d})$.

Turning now to the patterns taken with the beam parallel to the 3 axis, the sample radius (Figure 6) at small strains, two new features become apparent: (i) there is a marked improvement of order in the pattern (i.e. the arcs become sharper (compared with Figure 3b)); (ii) the two $\{10 \overline{1} 0\}$ reflections previously parallel to the sample surface (which we note is the plane of shear) remain in these positions but become stronger in comparison to the other four $\{10 \overline{1} 0\}$ reflections. With further strain, we see that this six-point pattern persists (Figure 6c) until finally at $\gamma=12$, the four weaker reflections disappear altogether and the pattern becomes a very sharp two-point pattern with reflections above and below the sample plane (Figure $6 \mathrm{~d}$ ).

Finally, in the case of the patterns taken with the beam parallel to the 1 axis, the circumferential direction of the sample (Figure 7), we see that after all strains the positions of maximum intensity lie above and below the sample plane. However, at small strain, we also see the appearance of two curious weak but sharp reflections at $90^{\circ}$ to the original reflections (Figure 7a,b). At higher strains (Figure 7c) we see evidence of a six-spot pattern developing with the two most intense arcs lying on the same position as before, on the normal to the sample plane, and the other four weaker reflections occurring at the positions consistent with hexagonal packing. At $\gamma=12$ (Figure 7d) this sixspot pattern is well developed and is clearly reminiscent of the sample pattern at very low strains, but with the beam parallel to the 3 axis, the sample radius (Figure 6a).

At this point it is important to note that, with increasing strain beyond $\gamma=12$ strain units, the order starts to deteriorate. Figure 8 illustrates the morphology at $\gamma=$ 18 , taken from a sample for which the maximum strain at the outer edge was 20 . With the beam along the 2 axis, the pattern shows an increase in the degree of arcing, indicating appreciable disorder compared to Figure $5 \mathrm{~d}$.
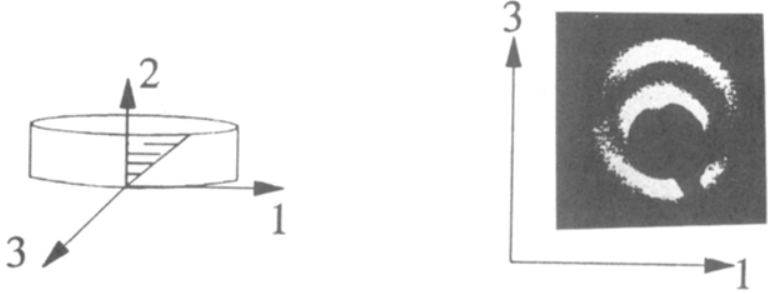

a)

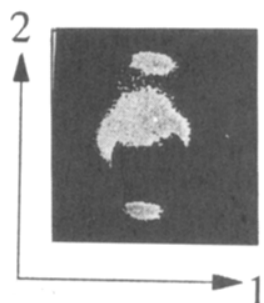

b)

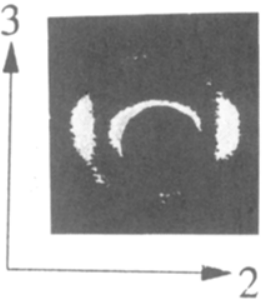

C)
Figure 8. SAXS patterns taken after a shear strain of $\gamma=18$. The sample was sheared with $\gamma_{R}=20$ in $25-\mathrm{mm}$ parallel plate geometry.

The SAXS pattern taken with the beam parallel to the sample radius, the 3 axis, is still two spot in nature with reflections occurring above and below the sample plane, quite similar to the pattern in Figure 6d. Finally, with the beam parallel to the flow direction, the 1 axis, the pattern is quite different from that obtained after $\gamma=12$ (Figure $7 \mathrm{~d})$. In this case, while there are still two reflections above and below the sample plane, the four other reflections previously visible in Figure 7d have now disappeared.

\section{Interpretation}

It is clear from both TEM and SAXS that the preparation method of planar extension has produced a great deal of orientation between neighboring grains such that the cylinders lie parallel to the extensional direction. However, despite this high degree of orientation, it is apparent from the electron micrographs that there remain grain boundaries in the material (Figure 4a). Indeed, the imperfect nature of the orientation is also apparent from the SAXS patterns, with all three projections showing appreciable disorder (Figure 3). The relative degrees of disorder are consistent with a strong tendency for cylinders to lie within the plane of the sheet. Again, in this initial morphology, it is also clear that the faces of the hexagons (the $\{10 \overline{10}\}$ planes) tend to align parallel to the sample surface.

After shearing and quenching, the structure still exhibits hexagonal symmetry and the $d$ spacings are unchanged. We are confident that the structure which exists during shear persists during the quenching to room temperature. From Figures $5 \mathrm{a}$ and $6 \mathrm{a}$, even after small shear, there is an increased randomization of the cylinders about the 2 axis, the sample normal. In Figure $5 \mathrm{a}$, with the beam parallel to the 2 axis, we see a greater degree of angular spread of the arcs and, in Figure $6 a$, with the beam parallel to the 3 axis, the sample radius, a decrease in the relative intensities of the four off-axis $\{10 \overline{1} 0\}$ reflections compared to those above and below the plane of the sample. This second effect is clearly a consequence of the increased disorientation of the hexagons around the 2 axis, moving the four off-axis $\{10 \overline{1} 0\}$ planes out of reflecting position while the $\{10 \overline{1} 0\}$ planes parallel to the sample surface 


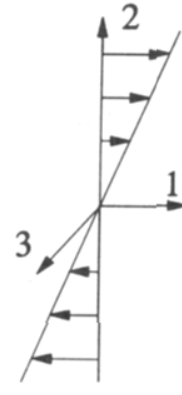

(a)

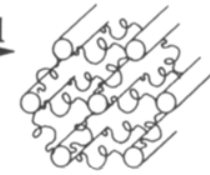

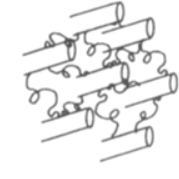

(b)
Figure 9. Schematic representation of the intermediate orientations that give rise to the two arcs and two spots in Figure $7 \mathrm{a}, \mathrm{b}$. The two components of the orientation are represented by (a) and (b).

remain in perfect reflecting position. (Of course, we recognize that all the cylinders were not perfectly parallel to the sample radius even in the unsheared state which may contribute to this effect but we assume that this influence is negligible.) The increased sharpness of the spots in Figure 6a also shows that after shear there is an increased tendency for the faces of the hexagons (i.e. $\{10 \overline{1} 0\}$ planes) to lie parallel to the plane of shear. Figure 7a, with the beam parallel to the 1 axis, the tangential view, shows two strong reflections above and below the sample plane as in the unsheared sample. Indeed, we expect these reflections to be particularly strong because we have seen a strong tendency for $\{10 \overline{10}\}$ planes to lie parallel to the sample plane and hence to be in perfect reflecting position when the beam is tangential. The curious two weaker spots, which comprise the other component of this pattern, clearly can only arise if there are $\{10 \overline{0} 0\}$ planes which are both parallel to the beam and perpendicular to the plane of the sample. We consider that the most probable situation which is consistent with these criteria is that portrayed schematically in Figure 9, where in (a) some grains are oriented with their cylinders in the plane of shear and in (b) a small fraction of grains is aligned with their cylinders parallel to the $(1,2)$ plane, but at an angle to the sample plane. Within this second component, where $\{10 \overline{10} 0\}$ planes are parallel to the $(1,2)$ plane, it is this population which gives rise to the weaker reflections in Figure 7a.

As the value of the applied shear strain was increased further, the essential features of the patterns remain the same but with increased disorder around the 2 axis, the sample normal. In fact, the pattern in Figure 5b appears almost isotropic while the radial (Figure 6b) pattern shows six spots and the tangential (Figure 7b) pattern exhibits two arcs and two spots. Again, there is a strong propensity for faces of the hexagons to lie parallel to the sample plane, apparent from Figures $6 \mathrm{~b}$ and $7 \mathrm{~b}$.

At this point it is useful to consider the electron micrograph of a section cut perpendicular to the sample radius, the 3 axis (Figure 10), from approximately the same position as the SAXS patterns of Figures $5 \mathrm{~b}, 6 \mathrm{~b}$, and $7 \mathrm{~b}$. Here we see that while the major proportion of the cylinders is essentially parallel to the radius, there is a component which we now see edge-on. This is clearly showing the presence of an intermediate morphology. Also, it appeared that the grain size was reduced as compared to the as-cast sample (Figure 2) and the unsheared sample (Figure 4a).

After slightly higher strain, $\gamma=7.8$, the pattern parallel to the 2 axis, the sample normal (Figure $5 \mathrm{c}$ ), indicates that the hexagonally packed cylinders are on the average slightly aligned in a tangential direction. The in-plane patterns are six-spot in nature (Figures $6 \mathrm{c}$ and $7 \mathrm{c}$ ), which

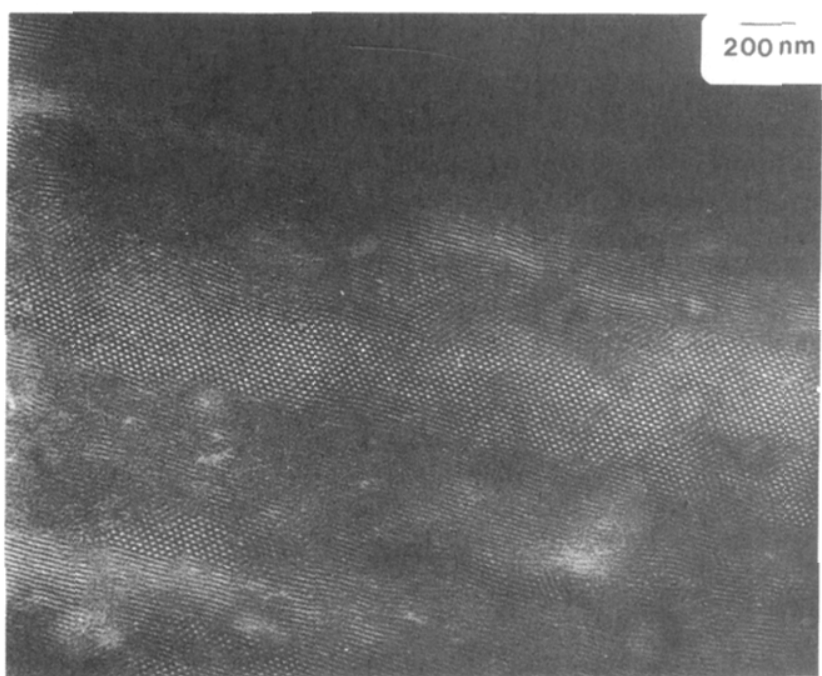

Figure 10. Electron micrograph from a cut perpendicular to the 3 axis, the sample radius at $\gamma=2.8$. There is a majority of cylinders lying end-on, indicating they are still parallel to the sample radius (initial order), and some are already lying more edge-on, indicating they are at an angle to the sample radius.

suggests that all the cylinders are now contained within the sample plane.

After a still higher strain of $\gamma=12.0$ (Figure 5d), the patterns taken along the 2 direction, the sample normal, show a further enhancement of cylinder orientation in the 1 direction. More dramatically, in the 3 direction, the radial projection, the transition from a six-spot to a twopoint pattern indicates that there is no longer a component of cylinders lying parallel to the 3 axis, the radius, and confirms the increased degree of alignment in the 1 direction, the flow direction. The six-point pattern in the 1 direction is entirely consistent with this, but with the additional information that there is still a strong tendency for $\{10 \overline{1} 0\}$ planes to be parallel to the sample plane. SAXS evidence is quite conclusive that the cylinders have realigned by $90^{\circ}$ into the direction of flow.

Above $\gamma=12$, the essential features of the patterns are all in reasonable agreement with the lower strain samples, but they are more disordered. The domain orientation starts to deteriorate. Figure $8 \mathrm{~b}$ shows only equatorial scattering consistent with the $\gamma=12$ sample, indicating that the cylinders are still contained within the sample plane. However, the pattern of Figure 8a, taken along the sample normal, shows a strong randomization at $\gamma=18$. The patterns taken parallel to the flow direction are consistent with an increase in randomization; see Figure $8 c$.

\section{Discussion}

The material was subjected to shear strains which are large enough to alter the morphology. One of the most striking features of flowing block copolymers is that the structure does not relax to its initial state as would usually happen in a strained rubber or a conventional polymer melt. This seems to be due to irreversible processes such as the breakup of grains or cylinders into smaller units, which has two effects. First, structural breakup would relieve some of the strain energy accumulated by the distorted lattice, and secondly, it would remove the memory of the original grain structure. After having been broken, these smaller units continue to be subjected to strain and rotate in the direction of flow. Block copolymers also have the remarkable ability to heal cracks and defects even at room temperature, as reported by Odell and 
Keller. ${ }^{17}$ We suggest that some of the same healing processes happen in our samples during cooling. However, because the memory of the original cylinder orientation has been substantially removed by the earlier cylinder/grain breakup, the cylinder orientation in the reformed grains does not revert to the original orientation, but rather corresponds to that in the material at the time of shear. Therefore, studying room-temperature patterns provides us with insight into the structural reorientation during shear even if it does not reveal the length scale of the broken structure.

The rheological properties of ordered SBS depend on direction, even in the molten state. ${ }^{14}$ The modulus is higher when shearing is normal to the cylinder direction than when shearing is in the direction of the cylinders. This suggests an energetic reason for the shear-induced alignment.

The alignment of cylindrical domains during shear depends not only on the amount of shear but also on the original orientation of individual domains. Despite our efforts to achieve a perfect single crystal type initial structure, there remained (i) some misalignment of cylinders within the sample plane and (ii) some out-of-plane misalignment. These two populations will respond differently to the applied shear field. By considering them separately, we can understand the development of the observed textures under shear flow conditions. The degree to which such alignment processes proceed depends critically upon the amount of applied shear.

Let us consider the effect of the flow field on the first population, the misaligned cylinders contained within the sample plane. Their behavior is affected by the curvature of the disk-shaped sample. When the shear is imposed, the ends of the cylinders at higher values of $r$ are subjected to a higher shear rate than the ends at lower $r$. This accelerates the alignment in the direction of flow. The rotation of the cylinders is strictly within the plane of shear.

Now consider the second population of cylinders which initially lie at an angle to the sample plane. In this case, the differential displacements will first cause the cylinders to rotate into the $(1,2)$ plane. Once they have reached this orientation, further shear will cause them to align parallel to the 1 axis.

By treating the system in terms of these two populations, we may visualize the evolving morphologies that are associated with the SAXS patterns. The flow may be stopped at any intermediate state, and the transient textures may be frozen in. Thus, we can readily understand the orientation process in the early stages of shear leading to the texture shown schematically in Figure 9. This twocomponent texture is achieved by (i) rotation of cylinders lying within the plane of shear toward the flow direction and (ii) the simultaneous rotation of cylinders to lie within the $(1,2)$ plane (tangential plane). Within this second component, there will be some grains which lie with $\{10 \overline{1} 0\}$ planes parallel to the $(1,2)$ plane and it is these which give rise to the weaker spots in the patterns in Figure 7a,b.

The morphological changes that accompany the transition in the SAXS pattern taken along the 1 axis from the four-point to six-point pattern, correspond to the stage at which the majority of this second component has rotated into the $(1,3)$ plane and is parallel to the X-ray beam. Eventually, we see that the 3 axis pattern changes from six-point to two-point character. This simply means that there are no longer any cylinders parallel to the 3 axis, the sample radius.
Beyond $\gamma=12$, we begin to see a deterioration in the degree of domain orientation. This effect was investigated in more detail elsewhere. ${ }^{18}$ The SAXS patterns indicate that the cylinders are still contained within the sample plane but are randomized about the flow direction. This increase in randomization can be attributed to the onset of cylinder breakup into shorter units, which then become increasingly more disordered. This is not a surprising result since the macromolecules are unable to accommodate the increased shear between domains and will simply be pulled out as the strain exceeds a certain value. The optimum strain for aligning the cylinders into the direction of flow with this deformation was found to be $\gamma=12$.

Most interesting is the observation that there is such a pronounced tendency for the $\{10 \overline{1} 0\}$ planes to lie parallel to the sample surface. The aligned cylinders become part of the shear surfaces of the imposed shear flow. An important parameter might be the spacing between shear surfaces which contain the cylindrical domains. The spacing is largest with the observed morphology, i.e. it would decrease for other orientations of the hexagon planes. This lets us assume that the large spacing is favored energetically. However, the effect might also be surface induced since it also occurred in planar extension (see Figure $3 \mathrm{~b}$ ), where the hexagonal planes aligned with the mold surface. More experiments are needed to clarify the origin of this phenomenon.

\section{Conclusions}

Shear flow introduces irreversible textural changes that are progressive with the amount of applied shear. This affects the direction of the cylindrical PS domains, the direction of the hexagonal planes, and the degree of order. With increasing strain, the order at first increases and then deteriorates. We set out to identify the early stages of structuring processes which occur in shear flow and to find an optimum strain for ordering.

The shear-induced morphology is retained by rapidly cooling the samples to room temperature. SAXS and TEM give sufficient information to propose models for the evolving domain orientation by analyzing intermediate states of orientation. The effect of a sufficiently large shear is to align cylinders parallel to the direction of shear. Hexagon planes align with the $(1,3)$ planes (shear planes). The proposed structuring process requires the cylindrical domains to break temporarily and heal again. However, the length scale of the broken structure could not be identified with the available techniques.

This study suggests that the processes during shear are highly influenced by initial morphology, and it would be informative to conduct analogous studies on samples of different starting textures.

Acknowledgment. H.H.W., D.S. and Y.-G.L. gratefully acknowledge the support of the Materials Research Laboratory (MRL) of the University of Massachusetts. F.E.K. and A.J.W. gratefully acknowledge the support of Grant AFOSR 91-001.

\section{References and Notes}

(1) Molau, G. In Colloidal and morphological behavior of block and graft copolymers. Block Copolymers; Aggarwal, S. L., Ed.; Plenum Press: New York, 1970.

(2) Helfand, E. Macromolecules 1975, 8 (4), 552.

(3) Keller, A.; Pedemonte, E.; Willmouth, F.M. Nature 1970, 225, 538; Kolloid Z. Z. Polym. 1970, 238, 385.

(4) Keller, A.; Dlugosz, J.; Folkes, M. J.; Pedemonte, F. P.; Scalisi, F. P.; Willmouth, F. M. J. Phys., Colloq. 1971, C5a, 295.

(5) Folkes, M. J.; Keller, A. Polymer 1971, 12, 222. 
(6) Dlugosz, J.; Keller, A.; Pedemonte, E. Kolloid Z.Z. Polym. 1970, 242,1125 .

(7) Arridge, R. G. C.; Folkes, M. J. Nature 1970, 5, 344; J. Phys. $D$ 1972, 5,344 .

(8) Hadziioannou, G.; Mathis, A. Colloid Polym. Sci. 1979, 257, 136.

(9) Lee, S. Ph.D. Thesis, L'Universite Louis Pasteur de Strasbourg, 1981.

(10) Weill, A.; Pixa, R. J. Polym. Sci., Polym. Symp. 1977, 58, 381.

(11) Terrisse, J. Ph.D. Thesis, L'Universite Louis Pasteur de Strasbourg, 1973.
(12) Morrison, F. A.; Winter, H. H. Macromolecules 1989, 22, 3533.

(13) Morrison, F. A.; Winter, H. H.; Gronski, W.; Barnes, J. D. Macromolecules 1990, 23, 4200.

(14) Scott, D. B.; Waddon, A.; Lin, Y. G.; Karasz, F.; Winter, H. H. To be submitted for publication to $J$. Rheol.

(15) Chatraei, Sh.; Macosko, C. W.; Winter, H. H. J. Rheol. 1981, $25(4), 433$.

(16) Khan, S. A.; Larson, R. G. Rheol. Acta 1991, 30 (1), 1-7.

(17) Odell, J. A.; Keller, A. Polym. Eng. Sci. 1977, 17, 544.

(18) Scott, D. B. Ph.D. Thesis, University of Massachusetts, 1992. 